\title{
Signalfunktion und Kodierungsgrad von sprachlichen Merkmalen in Gerichtsurteilen
}

\begin{abstract}
This article investigates the relations between the textual conventions of a text genre and the speech acts performed within this genre. The starting point of the study is a critical view upon Katharina Reiß' concept of "Textsortenkonventionen". It is stated that a mainly quantitative concept as Reiß' cannot adequately guide the translator in his search for the appropriate translation equivalent, and that a qualitative concept of textual convention has to be developed to fill this gap. As an example, an analysis of the speech act [report] within the text genre "legal judgements in Civil Law cases" is presented. As a result of the analysis, some of the conventions of the text genre are said to be coded signals for the speech acts performed within the genre. On the basis of these results an attempt is made to develop a theoretical concept that may describe the relation between degree of conventionalisation and the signal quality of linguistic elements within texts.
\end{abstract}

\section{Einleitung}

Der folgende Artikel ist der Versuch, Ansätze zu einem Konzept zu entwickeln, mit Hilfe dessen konventionell festgelegte Restriktionen bei dem Gebrauch sprachlicher Mittel in Landgerichtsurteilen und die Hintergründe solcher Restriktionen beschrieben werden können ${ }^{1}$. Ausgangspunkt ist die Hypothese, daß einige der in Gerichtsurteilen konventionell verwendeten sprachlichen Mittel Signale für sprachliche Handlungen sind. Als

1 Dieser Artikel ist ein Teilergebnis einer größeren Arbeit mit dem vorläufigen Titel "Grundlegung einer komparativen Analyse von syntaktischen und syntagmatischen Konventionen und ihrer Funktion in dänischen und deutschen juristischen Texten". Ich möchte mich bei Sven-Olaf Poulsen, Hans Kristian Mikkelsen und Henning Nølke, alle Aarhus, sowie bei Prof. Dr. Theo Bungarten, Hamburg für wichtige Hinweise und ertragreiche Diskussionen in Verbindung mit der Entstehung dieses Beitrages bedanken. 
Beschreibungsgrundlage versuche ich, einen Kodierungsgrad dieser Signale innerhalb einer Textsorte zu entwickeln. Der Kodierungsgrad ist ein Ausdruck dafür, wie stark die Konvention für z.B. die Angabe einer sprachlichen Handlung ist.

Die Kenntnis solcher Restriktionen und ihrer Hintergründe ist für Textproduzenten (und damit meines Erachtens auch für Übersetzer) nützlich. Nur ein Textproduzent, der solche Kenntnisse bewußt oder unbewußt besitzt (d.h. die entsprechende kommunikative Kompetenz besitzt), kann m.E. einen wirklich adäquaten Text verfassen. Für den Übersetzer bedeuten solche Kenntnisse u.a., daß er eine Auswahl treffen kann zwischen den sprachlichen Konventionen der Textsorte, die dem Zweck seiner Übersetzung dienen, und den Konventionen, bei denen dies nicht der Fall ist.

Eine solche Untersuchung von Textsortenkonventionen muß komparativ angelegt werden, um übersetzungsrelevant $\mathrm{zu}$ sein. Ideales komparatives Arbeiten erfordert ein einzelsprachenunabhängiges Tertium comparationis 2 . Nur mit einem solchen Vergleichsglied lassen sich sprachliche Elemente verschiedener Sprachen adäquat vergleichen. Um diesem Ideal so nahe wie möglich zu kommen, beschäftige ich mich in meiner komparativen Untersuchung mit Texten einer einzelnen Textsorte, deren Produktionssituation und kommunikative Zwecke klar zu umreißen und in den untersuchten Sprachgemeinschaften vergleichbar sind, nämlich mit Gerichtsurteilen. Diese Wahl ermöglicht es, kommunikative Funktionen einzelsprachenunabhängig zu beschreiben, und diese Funktionen können dann als Vergleichsbasis dienen. Was verglichen wird, sind somit die sprachlichen Mittel, die bei der Realisierung der kommunikativen Funktionen mitwirken.

Der Artikel besteht aus einem ersten Teil, in dem die Problemstellung erläutert wird und das Konzept der Signalfunktion behandelt wird. Danach folgt ein praktischer Teil, in dem die Analyse einer sprachlichen Handlung und ihrer Realisierung anhand eines Korpus von 25 deutschen und 19 dänischen Landgerichtsurteilen aus den Jahren 1988-90 präsentiert wird. Und abschließend wird das Konzept des Kodierungsgrades an dem vorgestellten Beispiel entwickelt.

$\overline{2}$ Fabricius-Hansen (1983), 28f. 


\section{Die Bedeutung der Textsortenkonventionen für die Kommunikation}

\subsection{Textsortenkonventionen nach Reiß}

Katharina Reiß hat in mehreren Publikationen den Begriff der Textsortenkonventionen (TSK) behandelt ${ }^{3}$. Sie versteht unter TSK die innerhalb einer Textsorte befolgten sprachlichen Konventionen auf allen Sprach- und Textebenen (Reiß/Vermeer 1984, 184 f). Es scheint ein rein statistisches Konzept zu sein: jedes sprachliche Merkmal, das häufig in Texten einer Textsorte auftritt, ist eine TSK. Reiß beschreibt die Funktion der TSK als die, Erkennungssignal für Textsorten zu sein. Mit Hilfe der TSK erkennt der Empfänger nach Reiß, um welche Textsorte es sich bei dem vorliegenden Text handelt. Aufgrund dieses Wissens rezipiert er den Text mit einer bestimmten Erwartungshaltung, und diese Erwartungshaltung beeinflußt und steuert das Textverstehen (Reiß/ Vermeer 1984, 189-191). Die Auffassung von Reiß ist folglich, daß TSK bei der Übersetzung berücksichtigt werden sollten.

Poulsen (1990) kritisiert mit Recht die Auffassung, daß alle TSK Textmerkmale sind, die für das Verständnis und die Kommunikation notwendig sind. Folglich ist sein Standpunkt, daß TSK bei der Übersetzung nicht automatisch zu befolgen sind. Nach Poulsen sollte "der Übersetzer [...] Textsortenkonventionen nicht ohne weiteres als Richtschnur akzeptieren, sondern sie nur dann befolgen, wenn sie ihm bei seiner Aufgabe als Mittler von Inhalten nützlich sind" (Poulsen 1990, 32). Das Befolgen einer Konvention bei der Übersetzung sollte somit von dem Zweck dieser Übersetzung abhängig sein.

Die Entscheidung, ob man eine Konvention bei der Übersetzung befolgen will oder nicht, setzt meines Erachtens jedoch ein umfassendes Wissen über die kommunikative Rolle der einzelnen Konventionen voraus. Bei manchen Textsorten wäre nämlich die fehlende Anwendung eines Teils der konventionell verwendeten sprachlichen Mittel genauso falsch wie die kritiklose Übernahme aller statistisch feststellbaren Konventionen einer Textsorte. Bei dem von Poulsen untersuchten Text (einer Bedienungsanleitung) sind die Grenzen der Auswahl weitgesteckt. Der

3 Z.B. Reiß/Vermeer (1984) und Reiß (1989). 
Übersetzer kann Struktur und sprachliche Mittel zur Ausfüllung der Struktur des Textes frei bestimmen, er muß nur zusehen, daß die notwendigen Instruktionen (= Funktion des Textes in diesem Fall) weitergegeben werden. Wie ich später zeigen werde, ist bei Gerichtsurteilen als institutionalisierter Kommunikation dagegen die Auswahl sowohl der Textstruktur als auch deren Ausfüllung stärker restringiert, sodaß die Textfunktionen nicht ohne Konsequenzen für die Kommunikation mit solchen sprachlichen Mitteln ausgeübt werden können, die nicht der Konvention entsprechen.

Das Problem besteht also darin, festzulegen, welche zielsprachlichen TSK mit den kommunikativen Funktionen verbunden sind, und bei welchen TSK die Konventionalität der sprachlichen Mittel auf andere Faktoren zurückzuführen sind. Die folgende erweiterte Hypothese ist der Versuch, an Hand der vorstehenden Überlegungen die TSK zu umschreiben, die für die Übersetzung von Landgerichtsurteilen von Bedeutung sind. Die Hypothese soll in diesem Artikel überprüft werden:

Ein Teil der TSK eines Textes sind kodierte Elemente, die als Signale für eine

sprachliche Handlung eine kommunikative Funkt

\subsection{Signalfunktion sprachlicher Merkmale}

Zunächst sei der Versuch gemacht, ausgehend von einem von Gutt (1991) eingeführten Konzept kommunikativer Signale einen theoretischen Rahmen für die Beschreibung der Rolle von TSK in der Fachkommunikation und ihrer Bedeutung für die Übersetzung zu schaffen.

Auf der Basis der Relevanztheorie von Sperber und Wilson (z.B. Sperber/Wilson 1986) versucht Gutt, eine einheitliche Erklärung für die kognitive Leistung "Übersetzen" zu entwickeln. Das Übersetzen wird grundsätzlich als eine Art der Kommunikation angesehen, bei der der Übersetzer sein Verständnis des zu übersetzenden Textes oder einen Teil davon je nach Übersetzungszweck in unterschiedlicher Weise weitervermittelt. Ist es bei der Übersetzung das Ziel des Übersetzers, einen Text zu schaffen, der sowohl das im Ausgangstext Gesagte als auch die linguistischen Eigenschaften des Ausgangstextes wiedergibt, dann spricht Gutt von "direkter Übersetzung" (Gutt 1991, 125-129). Die linguistischen Eigenschaften (z.B. Wortwahl, Wortstellung, phonetische Eigenschaften) werden als Mittel aufgefaßt, um Signale an den Empfänger zu geben, wie er den Text interpretieren soll. Gutt nennt diese Signale "communicative clues" 
(kommunikative Signale) (op. cit.127). Die Mittel, um kommunikative Signale geben zu können, lokalisiert er auf allen Ebenen der sprachlichen Kommunikation. Die Signalfunktion bei Gutt beruht auf der Anwendung ganz bestimmter Mittel. Ein Beispiel wäre das Erzielen einer ästhetischen Wirkung durch eine bestimmte Lautfolge. Bei der direkten Übersetzung eines kommunikativen Signals gilt es, die Funktion des kommunikativen Signals im Ausgangstext mit entsprechenden Mitteln im Zieltext auszuüben. Als weitere Beispiele für kommunikative Signale nennt er u.a. Verwendung von Wörtern mit bestimmtem Wert, Verwendung einer besonderen Wortstellung oder Verwendung formelhafter Ausdrücke (op. cit. 129-159).

Das Konzept der kommunikativen Signale ist weit differenzierter als das der TSK bei Reiß. Wo Reiß davon ausgeht, daß alle textsortenspezifischen linguistischen Eigenschaften eines Textes der Verständnissicherung durch Wiedererkennung der Textsorte dienen, geht Gutt davon aus, daß die einzelnen kommunikativen Signale unterschiedliche Funktionen in der Kommunikation ausüben (z.B. Fokussieren oder Erzielen einer ästhetischen Wirkung), und daß sie direkt (also nicht über die Erkennung der Textsorte) auf das Textverständnis des Empfängers wirken. Die Instanz der Textsorte hat damit bei Gutt einen weit geringeren Stellenwert beim Textverstehen als bei Reiß.

Mit der Auffächerung der Funktionen von konventionell festgelegten sprachlichen Mitteln in Bezug auf das Textverständnis ist es meines Erachtens möglich, Kriterien für eine Auswahl von Signalen zu erarbeiten, je nachdem welche Funktionen der Übersetzer zu übertragen hat. Damit sollte es auf der Grundlage dieses Ansatzes möglich sein, ein Konzept zu entwickeln, das die in Abschnitt 2.1 aufgestellte Hypothese bestätigen kann. Für die Untersuchung der Signalisierung von sprachlichen Handlungen in juristischen Texten nehme ich meinen Ausgangspunkt in dem, was Gutt kommunikative Signale aus formelhaften Äußerungen nennt (op. cit., 148152). Eines seiner Beispiele ist das englische Syntagma "Wet paint". Dieses Syntagma hat zwar einen logischen Eintrag im Sinne von Sperber/Wilson ${ }^{4}$,

4 Sperber/Wilson (1986) unterscheiden in ihrer Theorie über menschliche Kognition u.a. zwischen dem logischen Eintrag von Konzepten, der für die Erstellung einer vollständigen propositionalen Form durch den Empfänger notwendig ist, und dem enzyklopädischen Eintrag, der aus Informationen zur Entstehung und Umgebung des Konzeptes besteht (S. 86ff.). 
in dem die darin verwendeten Lexeme auf "nasse Farbe" verweisen. Die primäre Funktion des Syntagmas ist jedoch die, eine bestimmte sprachliche Handlung auszuüben, nämlich den Empfänger vor der noch nicht trockenen Farbe zu warnen. Das Syntagma wird bei dieser Handlung immer verwendet, was im enzyklopädischen Eintrag des Ausdruckes angelegt ist. Es wäre möglich, die sprachliche Handlung auch mit anderen Ausdrücken auszuführen, konventionell wird aber der erwähnte Ausdruck verwendet. Meines Erachtens existiert damit insofern eine Signalrelation zwischen dem Ausdruck und der sprachlichen Handlung, als daß der Ausdruck immer vorerst als die sprachliche Handlung interpretiert wird, ohne daß auf den logischen Eintrag zurückgegriffen wird. Will der Übersetzer die sprachliche Handlung im Zieltext wiedergeben, gilt es, in der Zielsprache ein Wort, ein Syntagma oder ein sonstiges sprachliches Element zu finden, das dieselbe Signalfunktion innehat. Im Deutschen wäre das Äquivalent das Syntagma "Frisch gestrichen", im Dänischen "Nymalet"5.

Sinn der Verwendung des Signals ist es also, anzugeben, daß eine bestimmte sprachliche Handlung ausgeübt wird. In dieser besonderen Verwendung ist der logische Eintrag verblaßt und das Syntagma sozusagen zu einem kodierten Element erstarrt ${ }^{6}$. Beim obigen Beispiel handelt es sich um einen Kode, der Teil der normalen muttersprachlichen Kompetenz ist. Jedoch ist es auch denkbar, daß bestimmte Elemente (TSK) nur bei Verwendung in besonderen Kommunikationssphären wie z.B. der Urteilskommunikation eine kodierte Signalfunktion haben. In allen Fällen ist die Einhaltung der Konventionen notwendig, wenn (nach der Terminologie von Gutt, s.o.) eine direkte Übersetzung der sprachlichen Handlung zustandekommen soll, und Stolze (im Druck) weist darauf hin, daß diese Art der Übersetzung bei Urteilen in der Praxis die Norm ist.

\section{Analyse von Gerichtsurteilen}

$5 \mathrm{Zu}$ einem ähnlichen Ansatz, jedoch auf der Grundlage der "Speech Act Theory", siehe Kußmaul (1990).

6 Anscombre (1985) hat die Änderung der Bedeutung eines Wortes von primär propositioneller Bedeutung hin zu einer Bedeutung, die primär auf die mit dem Wort ausgeführte illokutionäre Handlung fußt, als "délocutivité" beschrieben. Er versteht das Phänomen als eine Art Derivation (S. 172f). 


\subsection{Erarbeitung der Vergleichsgrundlage}

Um die anfangs genannten einzelsprachenunabhängigen Funktionen zu erschließen, habe ich versucht, theoretisch-deduktiv die pragmatischen Bedingungen von Landgerichtsurteilen zu erarbeiten 7 . Daraus ergibt sich, daß der Sender des Urteils das erkennende Gericht ist und daß das Urteil an vier verschiedene Kategorien von Empfängern gerichtet ist. Für jeden Empfänger läßt sich eine andere Senderintention formulieren, obwohl derselbe Text in allen Kommunikationssituationen verwendet wird. Das Ergebnis der Analyse ist der folgenden Figur zu entnehmen:

\section{Figur 1}

$\mathrm{Zu}$ jeder Kommunikationssituation gehören verschiedene sprachliche Handlungen, mit denen der Sender versucht, die jeweilige Senderintention zu verwirklichen. Diese sprachlichen Handlungen werden nach der Theorie

$7 \quad$ Engberg (1992b). Die Analyse bezieht sich speziell auf das deutsche Landgerichtsurteil, frühere Arbeiten haben aber ergeben, daß deutsche und dänische Landgerichtsurteile sich bezüglich der pragmatischen Bedingungen so stark ähneln, daß eine Analyse des einen Typs auch für die andere gilt (Engberg (1990), 18f). Zur Analysemethode siehe Weber (1991), bes. S. 270f. 
sprachlicher Handlungen von Weber u.a. ${ }^{8}$, die ich als Grundlage meiner Arbeit verwende, durch konventionell festliegende sprachliche Mittel (TSK) ausgeübt. Wenn der Übersetzer weiß, welche Senderintentionen er aufgrund des Übersetzungszweckes in den Zieltext zu übertragen hat, kann er folglich diejenigen zielsprachlichen TSK auswählen, die er zu befolgen hat, um die gewünschte Senderintention konventionell richtig auszudrücken.

Den Ausführungen in diesem Artikel lege ich die Aufgabe zugrunde, Urteile für den Gebrauch in Verbindung mit der Rechtsdurchsetzung im Ausland zu übersetzen. Damit ein ausländisches Urteil z.B. in Deutschland anerkannt und vollstreckt werden kann, muß ein deutsches Gericht darüber befinden, ob das ausländische Urteil seinem Inhalt nach mit einem deutschen Urteil oder Verfahren oder mit wesentlichen Grundsätzen des deutschen Rechts (§ 328 I Ziff. 3 und 4 ZPO) unvereinbar ist. Diese Prüfung erfolgt auf der Grundlage der Übersetzung. Empfänger einer solchen Übersetzung sind somit Richter (und aufgrund des Rechts von Bürgern auf Rechtsbeistand auch Anwälte) im Ausland, und die zu übertragenden Funktionen sind die, die bei der Beurteilung der Rechtsprechung wichtig sind.

\subsection{Exemplarische Analyse einer Sprachhandlung in Urteilen}

Den Teiltexten von Urteilen können sprachliche Handlungen zugeteilt werden. Figur 2 zeigt die Verteilung der übergeordneten sprachlichen Handlungen auf die vier Teiltexte ersten Grades ${ }^{9}$ des Urteils ${ }^{10}$ :

Fig. 2

Rubrum (dä: "Hoved"):

[Bedingungen für deklarativen Sprachgebrauch schaffen]

8 Siehe z.B. Weber (1991); zu einer Analyse von einer sprachlichen Handlung in Landgerichtsurteilen auf der Grundlage dieser Theorie siehe auch Engberg (1992b).

9 Nach der Terminologie von Gülich/Raible ist der Text als Ganzes in Teiltexte 1. Grades eingeteilt, die wiederum in Teiltexte 2. Grades eingeteilt sein können, usw. (Gülich/Raible 1975,159, Anm. 20).

10 Zur Einteilung des Urteils in vier Teiltexte, siehe Altehenger (1981); aus Platzgründen wird die Bestimmung der Anforderungen in dieser Arbeit nicht dokumentiert. Für eine begründete Zuschreibung der Sprachhandlungen zu drei Kommunikationssituationen, die die vorläufige Grundlage der Analysearbeit bildet, siehe Engberg (1992a). Die vollständige Dokumentation erfolgt in einer späteren Arbeit. 
Tenor (dä: "Domsformular"):

[Das Urteil fällen]

Tatbestand (dä.: "Sagsfremstilling"):

[Das zu lösende Problem präsentieren und juristisch festlegen]

Gründe (dä.: "Præmisser"):

[Die Entscheidung begründen]

Die übergeordneten Sprachhandlungen können innerhalb der Teiltexte durch untergeordnete Sprachhandlungen realisiert werden. So ist die im folgenden $\mathrm{zu}$ untersuchende Sprachhandlung [Referieren] eine der untergeordneten Sprachhandlungen, die innerhalb des Teiltextes "Tatbestand" (dä: "Sagsfremstilling") der Realisierung von [Das zu lösende Problem präsentieren und juristisch festlegen] dienen.

Untersucht wird die Art, wie [Referieren] in den Teiltexten zweiten Grades "Vortrag" (dä.: "Anbringender") erfolgt. Es handelt sich in diesen Teiltexten um die Wiedergabe der Angriffs- und Verteidigungsmittel des Klägers und des Beklagten. Es handelt sich somit um zwei Teiltexte, die aber in meiner Arbeit wegen ihrer formalen Einheitlichkeit zusammen behandelt werden.

Der ganze Teiltext "Tatbestand" ist eine Wiedergabe von Informationen über das zu lösende Problem, das dem Gericht vorgelegt worden ist. Sowohl im deutschen als auch im dänischen Zivilprozeß gilt die Verhandlungsmaxime, nach der "die Parteien bestimmen (...), welchen Prozeßstoff das Gericht seiner Entscheidung zugrundezulegen hat. (...) Tatsachen, die unter den Parteien unstreitig sind, müssen vom Gericht als wahr hingenommen werden" (Heintzmann 1985, 10). Der Tatbestand als Wiedergabe des zu lösenden Problems besteht somit aus zwei Arten der Information:

1)Themen, über die zwischen den Parteien Einigkeit herrscht

(Sachstand).

2)Themen, über die gestritten wird (Streitstand).

Da wie gesagt nur die Themen des Streitstandes Gegenstand der Entscheidung des Richters sein dürfen, ist die Unterscheidbarkeit der Informationstypen im Urteil wichtig. Deshalb benutzt der Richter die Sprachhandlung [Feststellen von Sachverhalten], wenn er den Sachstand 
wiedergibt. Typisch für diese Sprachhandlung ist, daß das Wiedergegebene als Faktum dargestellt wird und daß kein anderer Urheber der Information außer dem Sender präsentiert wird.

Wird dagegen der Streitstand wiedergegeben, benutzt der Richter die Sprachhandlung [Referieren]. Charakteristisch für diese Sprachhandlung ist, $\mathrm{da} ß$ die Äußerung eines anderen wiedergegeben wird und daß diese Äußerung immer als die eines anderen gekennzeichnet wird. Damit diese beiden Sprachhandlungen mit Sicherheit auseinandergehalten werden können, sind einige sprachliche Mittel notwendig, die den unterschiedlichen Charakter der Sprachhandlungen signalisieren.

\subsection{1. [Referieren] im deutschen "Vortrag"}

\section{Beispiele:}

\section{Beispiel 1}

Die Kläger tragen vor:

Sie hätten sich vor dem Erwerb ausdrücklich nach der Schallisolation erkundigt

und darauf hingewiesen, ...

\section{Beispiel 2}

Der Kläger behauptet, der Beklagte sei seit seiner Gründung im Jahre 1937

Im "Vortrag" wird das Referierte immer im Konjunktiv wiedergegeben. Dieser Konjunktiv wird immer von mindestens einem Äußerungsverb pro Vortrag eingeleitet 11 . Subjekt des ersten Äußerungsverbes ist entweder Kläger oder Beklagter. Bei eventuell folgenden Äußerungsverben kann entweder wie oben die Prozeßrolle oder das entsprechende persönliche Pronomen Subjekt sein.

In 5 von 25 untersuchten Urteilen kommt nur ein Äußerungsverb am Anfang des Vortrages jeder Partei vor (siehe Beispiel 1). In den restlichen Urteilen sind mehrere Äußerungsverben in jedem Vortrag vorhanden, und diese kommen auch häufig innerhalb eines typographischen Abschnittes vor (Beispiel 2). Es scheint eine Tendenz vorzuliegen, Referate, bei denen Auffassungen zum Sachverhalt dargestellt werden, durch bestimmte

$11 \mathrm{Zu}$ einer generellen Untersuchung des Tempus- und Modusgebrauchs in Landgerichtsurteilen siehe Harboe/Poulsen (1983). 
Äußerungsverben (z.B. behaupten und geltend machen) einzuleiten, wogegen Referate von Rechtsauffassungen durch andere Äußerungsverben bzw. Funktionsverbgefüge (z.B. Der Ansicht sein und meinen) eingeleitet werden. Diese Anwendung der Äußerungsverben ist aber nicht bei allen Urteilen vorhanden In der größeren Arbeit werde ich diese Tendenz näher untersuchen.

\subsection{2. [Referieren] im dänischen "Anbringender"}

\section{Beispiel}

Sagsøgte har endvidere anf $\phi r t$, at beslutningen om aktieudvidelsen lovligt er

truffet i henhold til aktieselskabslovens $§ 37$. Da 1

Sagsøgte har til støtte for sine påstande anført, at der ikke er noget

ansvarsgrundlag, idet sagsøgtes forsikringstager ikke har udvist noget

erstatningspådragende forhold, ...

Im dänischen Teiltext gibt es keine Verbindung zwischen der Wahl der Äußerungsverben und dem Inhalt des Referats (Sachverhalts- oder Rechtsauffassungen). Am Anfang eines jeden Vortrages befindet sich ein Äußerungsverb. Es können weitere Äußerungsverben vorkommen, meistens, um den Übergang von einem Klagepunkt zum anderen oder vom Angriff in die Verteidigung zu markieren. Subjekt ist die Prozeßrolle (Sagsøger/Sagsøgte) oder (bei späteren Äußerungsverben) das entsprechende persönliche Pronomen.

Das Referierte steht im Gegensatz zum deutschen Vortrag normalerweise im selben Tempus wie die ursprüngliche Äußerung. Tempusverschiebung kommt in meinem Korpus nicht vor, obwohl dies eine Möglichkeit des dänischen Sprachsystems ist, um Referat anzuzeigen 12 .

Dem folgenden Schema sind die Auswahl der Äußerungsverben sowie die Zahl der Urteile, in denen sie vorkommen, zu entnehmen. Bei den deutschen Verben ist der eingeleitete Referattyp (Sachverhalte (SV) oder Rechtsauffassung (R)) angegeben:

$\begin{array}{llrlr}\text { behaupten } & (\mathrm{SV}) & 16 / 25 & \text { gøre gældende } & (16 / 19) \\ \text { geltend machen } & (\mathrm{SV}) & 6 / 25 & \text { anføre } & (15 / 19) \\ \text { vortragen } & (\mathrm{SV}) & 5 / 25 & \text { bestride } & (4 / 19)\end{array}$

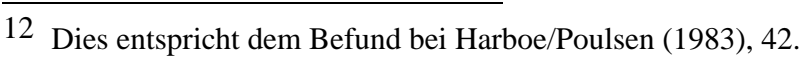




$\begin{array}{lllll}\text { bestreiten } & (\mathrm{SV}) & 4 / 25 & \text { fremhæve } & (1 / 19) \\ \text { ansehen als } & (\mathrm{SV}) & 2 / 25 & \text { finde } & (1 / 19) \\ \text { einwenden } & (\mathrm{SV}) & 1 / 25 & & \\ \text { entgegnen } & (\mathrm{SV}) & 1 / 25 & \\ \text { erwidern } & (\mathrm{SV}) & 1 / 25 & \\ \text { hinweisen auf } & (\mathrm{SV}) & 1 / 25 & \\ \text { sich berufen auf } & (\mathrm{SV} / \mathrm{R}) & 2 / 25 & \\ \text { der Ansicht sein } & (\mathrm{R}) & 11 / 25 & \\ \text { meinen } & (\mathrm{R}) & 10 / 25 \\ \text { der Auffassung sein } & (\mathrm{R}) & 3 / 25 \\ \text { die Auffassung vertreten } & (\mathrm{R}) & 3 / 25 \\ \text { ausführen } & \text { (R) } & 2 / 25 \\ \text { bezweifeln } & \text { (R) } & 1 / 25 \\ \text { folgern } & \text { (R) } & 1 / 25 \\ \text { der Meinung sein } & \text { (R) } & 1 / 25\end{array}$

\section{TSK als kodierte Elemente}

Auf der Basis der vorgestellten Analysen sollen die behandelten TSK näher beschrieben und kategorisiert werden. Meine Hypothese ist wie im Abschnitt 2.1 angeführt, daß die im vorigen Abschnitt beschriebenen TSK kodierte Elemente sind, die auf die vorliegende Sprachhandlung hinweisen und damit die Interpretation der darauf folgenden Information bei einem Kenner des Kodes (einem fachsprachlich gebildeten Empfänger) steuern. Für einen Kode ist normalerweise typisch, daß zwischen Kodeelement und dem, wofür das Kodeelement steht, eine 1:1-Entsprechung besteht. Wie aus dem Beispiel hervorgeht, ist dies aber bei den untersuchten TSK nicht der Fall. Kein einzelnes Element verweist alleine auf die Sprachhandlung [Referieren]. Im Deutschen geschieht dies durch den Konjunktiv in Verbindung mit einem Äußerungsverb oder einem entsprechenden Wortgefüge aus einer breiten Palette von Möglichkeiten, im Dänischen durch eines von wenigen Äußerungsverben.

Es ist somit notwendig, ein skaläres Beschreibungsmodell der Relation zwischen sprachlicher Handlung und sprachlichem Mittel zu benutzen. An dem einen Ende der Skala liegt die 1:1-Entsprechung und am anderen Ende die 1:unendlich vielen-Relation. Entscheidend für die Notwendigkeit, als Textverfasser genau ein bestimmtes sprachliches Mittel in einer bestimmten Situation zu verwenden, ist die Stärke der Konvention, verstanden als die Zahl der sprachlichen Mittel, die konventionell korrekt verwendbar sind. Bei 
TSK, die Sprachhandlungen ausüben und die damit als Signale für die Kommunikation von Bedeutung sind, nenne ich diese Stärke ihren Kodierungsgrad.

Für den höchsten Kodierungsgrad ist typisch, daß ein Element nur eine bestimmte Sprachhandlung ausführen kann und daß nur dieses bestimmte Element die Sprachhandlung ausführen kann (1:1-Relation). Es ist in der Kommunikation mit Benutzern des Kodes (in meinem Falle Juristen) notwendig, gerade dieses besondere sprachliche Mittel (das kodierte Element) zu benutzen, da die Fachleute nur das kodierte Element als Signal der Sprachhandlung erwarten. Die Verwendung eines anderen sprachlichen Mittels wäre für die Kommunikation störend, da der Empfänger es zuerst nicht als Signal der gemeinten Sprachhandlung, sondern als ein Element mit anderer Bedeutung interpretieren würde. Dies gilt z.T. für die Verwendung des Konjunktivs bei der deutschen Realisierung der Sprachhandlung [Referieren] im "Vortrag", jedoch mit der obengenannten Einschränkung, daß der Konjunktiv immer in Verbindung mit mindestens einem auslösenden Element (Äußerungsverb oder dergleichen) am Anfang des Vortrages auftreten muß und damit nicht alleine die Sprachhandlung signalisieren kann 13

Je mehr sprachliche Mittel als Signal für eine Sprachhandlung möglich sind, desto niedriger ist der Grad der Kodierung und desto schwächer die TSK (und je schwächer folglich auch der Zwang, einer bestimmten Konvention bei der Übersetzung zu folgen). So ist die Auswahl der möglichen Äußerungsverben jedenfalls innerhalb meines begrenzten Korpus im Deutschen viel größer als im Dänischen, und damit ist die Konventionalisierung der Äußerungsverben wegen der geringeren Auswahlmöglichkeiten im Dänischen stärker als im Deutschen. Anzumerken wäre noch, daß es sich hier nicht um eine direkt quantifizierbare Größe handelt, sondern um eine relative: es läßt sich kaum mit endgültiger Sicherheit festlegen, ob es fünf, sechs oder zehn Äußerungsverben gibt, die in dänischen Urteilen verwendbar sind. Aber schon die hier präsentierte Untersuchung deutet an, daß es bei deutschen Urteilen sehr viel mehr Möglichkeiten als bei dänischen Urteilen gibt ${ }^{14}$.

Eine mögliche Erklärung für diesen Unterschied liegt in der Funktion der untersuchten TSK. Wie dargelegt, enthält die Urteilskommunikation Signale,

\footnotetext{
13 Der Konjunktiv ist generell im Deutschen kein obligatorisches Mittel zur Kennzeichnung von indirekter Rede (Helbig/Buscha (1986), 195). Daß er bei dieser Textsorte obligatorisch ist, spricht für seine Kodiertheit.

14 Zur Adäquatheit von komparativen gegenüber klassifikatorischen Begriffen bei der Beschreibung menschlicher Kognition siehe Sperber/Wilson (1986), $79 \mathrm{f}$.
} 
die eine Differenzierung zwischen Sachstand und Streitstand ermöglichen und den Urheber der referierten Äußerungen angeben. Sowohl in dänischen als in deutschen Urteilen werden die Referate durch einen Satz eingeleitet, dessen Verbal ein Äußerungsverb oder ein ähnliches Element ist (siehe oben). Das Verb gibt also an, daß jemand etwas geäußert hat. Durch das Subjekt dieses Einleitungssatzes wird der Urheber dieser Äußerungen angegeben. Im deutschen Urteil wird der Unterschied zwischen Sach- und Streitstand auch durch den bei der Sprachhandlung [Referieren] obligatorischen Konjunktiv im Referat angezeigt. Im Dänischen wird diese Funktion nur durch das Äußerungsverb ausgeübt. Weiter muß das dänische Äußerungsverb noch eine dritte Funktion ausüben. Im dänischen Urteil werden außer den "Anbringender" auch Zeugenaussagen und Aussagen von Sachverständigen wiedergegeben. Folglich müssen die Referate von "Anbringender" so gekennzeichnet werden, daß sie sich von den übrigen Referaten in "Sagsfremstilling" unterscheiden. Im deutschen Urteil werden Zeugenaussagen und Aussagen von Sachverständigen nicht im "Tatbestand" referiert. Stattdessen befinden sich diese im Anhang des Urteils, worauf im "Tatbestand" verwiesen wird. Die Funktion, unterschiedliche Arten von referierten Aussagen voneinander zu unterscheiden, ist somit im deutschen Urteil nicht erforderlich.

Die Häufung der Funktionen, die mit ein und demselben Element ausgeübt werden, könnte ein Grund für die recht starke Konventionalisierung der Auswahl von Äußerungsverben bei dänischen Urteilen und der freieren Auswahl bei deutschen Urteilen sein. Wie gesagt hat das deutsche Äußerungsverb im "Vortrag" wegen des obligatorischen Konjunktivs kommunikativ primär die Funktion, durch sein Subjekt den Urheber des Referierten zu kennzeichnen. Deshalb scheinen jedenfalls sehr viele, wenn nicht alle Äußerungsverben den Platz ausfüllen zu können, obwohl einige häufiger gewählt werden als andere 15 . Damit ist es möglich, durch die Wahl des Âußerungsverbs mehr als nur die eine, kommunikativ erforderliche Funktion auszuüben. Oben wurde dargestellt, daß im deutschen Vortrag darauf hingewiesen werden kann, ob das Referat eine Rechts- oder Sachverhaltsauffassung betrifft. Weiter sind aber auch Markierungen der argumentativen Rolle der Ausführungen durch die Wahl eines entsprechenden Verbs möglich (z.B. erwidern, folgern, einwenden, bezweifeln, entgegnen).

Solche Markierungen sind dagegen mithilfe der Äußerungsverben in dänischen Urteilen kaum möglich. Ihre Bedeutung erschöpft sich im Ausdrücken der oben dargestellten komplexen fachsprachlichen

15 In Kjær (1990) werden solche Konventionen der Routine innerhalb eines Fachgebietes zugesprochen. Sie sind relativ schwach normiert (S. 95-104). 
Kodebedeutung, und die Zahl der konventionell korrekt verwendbaren Verben ist gering. Der logische Eintrag dieser Äußerungsverben scheint verblaßt zu sein, und dafür hat der enzyklopädische Eintrag an Bedeutung gewonnen. Wegen ihrer Spezialisierung sind sie kaum mit anderen Äußerungsverben austauschbar. Die konventionell verwendeten Äußerungsverben können deshalb als hochgradig kodierte kommunikative Signale beschrieben werden, die wegen ihrer Bedeutung für die Kommunikation bei der Übersetzung befolgt werden sollten. Z.B. müßte jedenfalls auf der Grundlage meines begrenzten Textkorpus die deutsche Redeeinleitung "Der Beklagte bezweifelt, daß ..." meines Erachtens mit "Sagsøgte har anført, at det er tvivlsomt, om ..."16 übersetzt werden, um den dänischen Konventionen $\mathrm{zu}$ entsprechen. Untersuchungen im Rahmen meiner weiteren Arbeit sollen klarstellen, wie häufig solche Einstellungsmarkierungen sind, und wie sie gegebenfalls in den beiden Sprachen markiert werden.

Zusammenfassend können folgende Thesen zum Konzept des Kodierungsgrades aufgestellt werden:

1) Die Rekurrenz eines sprachlichen Merkmals innerhalb einer Textsorte (Textsortenkonvention) kann direkt auf die Rekurrenz einer sprachlichen Handlung bezogen sein. In solchen Fällen tritt das sprachliche Element als kodiertes Signal für die sprachliche Handlung auf.

2) Je weniger sprachliche Elemente als Ausdruck einer sprachlichen Handlung möglich sind, desto stärker ist die Signalfunktion des einzelnen sprachlichen Elements.

3) Je stärker die Signalwirkung eines sprachlichen Elements ist, desto weniger austauschbar ist dieses Element.

\section{Ausblick}

Ich habe in diesem Artikel versucht, den Nutzen eines solchen Konzeptes für die Übersetzung darzustellen. Auf der Basis weiterer Analysen von Sprachhandlungen und ihren fachsprachlichen Signalen nach dem hier vorgestellten Muster sollte es meines Erachtens möglich sein, zu einer adäquaten Beschreibung der Textsorte Landgerichtsurteil zu kommen. Eine solche Beschreibung kann für die Übersetzung nützlich sein, weil sie die Mittel aufzeigt, mit denen verschiedene Funktionen in verschiedenen Sprachen ausgeübt werden.

\footnotetext{
16 Wörtlich übersetzt: "Der Beklagte hat behauptet, daß es zweifelhaft ist, ob ...".
} 
Aber auch für den mutter- oder fremdsprachlichen Fachsprachenunterricht ist eine solche Beschreibung potentiell von Nutzen. Die Kenntnis der kodierten Bedeutung der verschiedenen Signale ist auch bei der Rezeption von fachsprachlichen Texten notwendig. Diese Kentnisse können natürlich über Erfahrung, d.h. durch das Lesen vieler Fachtexte, intuitiv erlangt werden. Ökonomischer ist aber die Vermittlung dieser Kenntnisse im Unterricht, sofern sie gesichert vorliegen. Und gerade in diesen Jahren scheinen Linguisten dieses Arbeitsfeld der angewandten Forschung für sich zu entdecken. Ein Beispiel ist die Arbeit von Martina Hinze (1988) zu Rezensionen in Fachzeitschriften. Ein anderes Beispiel ist die von Gnutzmann/Oldenburg (1991) präsentierte Untersuchung von Struktur und Strukturierungsmitteln in englischen und deutschen wissenschaftlichen Artikeln.

Eine Beschränkung des hier vorgestellten Verfahrens, das die Kommunikationsintention des Senders als Grundlage nimmt, möchte ich jedoch zum Schluß noch erwähnen. Das Verfahren kann Strukturen und konventionell verwendete Mittel erfassen, die mit den im Text ausgeübten sprachlichen Handlungen verbunden sind, und kann damit den Rahmen eines Textes oder Teiltextes erklären. Aber die Analyse ist auf die Vertextungsverfahren beschränkt, bei denen eine wiederkehrende Prozeßhandlung oder dergleichen im Text abgebildet wird. Sobald die Individualität des Gerichtsprozesses überwiegt, müssen andere Verfahren herangezogen werden 17 . In seiner jetzigen Form kann das hier von mir entwickelte Konzept begründete Ratschläge dafür geben, wie sprachliche Handlungen in Urteilen gestaltet werden sollten. Für die Formulierung dessen, was z.B. referiert wird, kann das Verfahren aber jedenfalls zur Zeit keine Ratschläge geben.

\section{Literatur}

Altehenger, Bernd (1983): Die richterliche Entscheidung als Texttyp. In: Petöfi, Janos S. (Hrsg.): Texte und Sachverhalte (= Papiere zur Textlinguistik 42), Hamburg 1983, S. 185 227.

Anscombre, Jean-Claude (1985): Onomatopées, délocutivité et autres blablas. In: Revue Romane 20,2, København, S. 169-206.

Brinker, Klaus (1988): Linguistische Textanalyse, 2. Aufl., Berlin 1988.

Engberg, Jan (1990): Har tekstsortkonventioner en kommunikativ baggrund?, Diplomarbeit, Wirtschaftsuniversität Århus.

17 Siehe hierzu auch Stolze (im Druck). 
Engberg, Jan (1992a): Wie analysiert man Gerichtsurteile? Ein Plädoyer für eine textsortenspezifische Textanalyse. In: Grindsted, Anette/ Johannes Wagner (Hrsg.): Communication for Specific Purposes (= Kommunikation und Institution, Bd. 21). Tübingen 1992.

Engberg, Jan (1992b): Textanalyse von juristischen Fachtexten auf kommunikativer Grundlage - Methodologische Überlegungen an einem Analysebeispiel. In: Bungarten, Theo (Hrsg.): Beiträge zur Fachsprachenforschung. Sprache in Wissenschaft und Technik, Wirtschaft und Rechtswesen (= HAFF. Hamburger Arbeiten zur Fachsprachenforschung, Bd. 1). Attikon Verlag, Tostedt 1992.

Fabricius-Hansen, Cathrine (1983): Om såkaldt direktional og adirektional kontrastering. In: Kontrastiv Lingvistik i Norden, København, S. 28-33.

Gnutzmann, Claus/ Hermann Oldenburg (1991): Contrastive Text Linguistics in LSPResearch: Theoretical Considerations and some Preliminary Findings. In: Schröder, Hartmut (Hrsg.): Subject-oriented Texts, Berlin - New York 1991, S. 103-136.

Gülich, Elisabeth/ Raible, Wolfgang (1975): 'Textsorten - Probleme'. In: Linguistische Probleme der Textanalyse (= Jahrbuch des Instituts für deutsche Sprache 1973). Düsseldorf: Schwann 1975.

Gutt, Ernst-August (1991): Translation and Relevance, Oxford - Cambridge, MA: Basil Blackwell 1991.

Harboe, Eva/ Sven-Olaf Poulsen (1983): Hovedtendenser vedrørende tempus- og modusbrugen i danske og tyske domme i 1. instans, (= ARK 17), København.

Hintze, Martina (1988): Linguistische Untersuchung deutschsprachiger Rezensionen überwissenschaftliche (insbesondere technikwissenschaftliche) Publikationen, Chemnitz (Dissertation).

Kjær, Anne Lise (1990): Normbetingede ordforbindelser $i$ tysk juridisk sprog, (ARK 56), København.

Kußmaul, Paul (1990): Die Übersetzung von Sprechakten in Textsorten. In: Der Deutschunterricht 1/90, S. 17-22.

Poulsen, Sven-Olaf (1990): Zur Problematik des textsortenbezogenen Übersetzens. In: Der Deutschunterricht 1/90, S. 29-35.

Reiß, Katharina (1989): Textsortenkonventionen und Übersetzen. In: Hermes 2-89; 37-54.

Reiß, Katharina/ Vermeer, H.J. (1984): Grundlegung einer allgemeinen Translationstheorie, Tübingen: Niemeyer 1984.

Searle, John R. (1976): A classification of illocutionary acts. In: Language in Society, vol. 5, 1976, S. 1-23.

Seibert, Thomas-Michael (1981): Aktenanalysen. Zur Schriftform juristischer Deutungen, Tübingen: Gunter Narr Verlag 1981.

Sperber, Dan/ Wilson, Deirdre (1986): Relevance - Communication and Cognition, Cambridge, MA: Harvard University Press 1988. 
Stolze, Radegundis: Rechts- und Sprachvergleich beim Übersetzen juristischer Texte. In: Baumann, Klaus-Dieter/ Hartwig Kalverkämper (Hrsg.): Kontrastive Fachsprachenforschung (= Forum für Fachsprachenforschung, 20), Tübingen: Narr (im Druck).

Weber, Siegfried (1991): Communicative Acts and the Constitution of Scientific and Technical Texts. In: Schröder, Hartmut (Hrsg.): Subject-oriented Texts, Berlin - New York 1991, S. 267-303. 